MIT-CTP-3320

BUHEP-02-38

\title{
Analysis of a Toy Model of Electron "Splitting"
}

\author{
B. Altschul| \\ Department of Mathematics \\ Massachusetts Institute of Technology \\ Cambridge, MA, 02139-4307 USA \\ C. Rebbi \\ Department of Physics \\ Boston University \\ Boston, MA, 02215 USA
}

\begin{abstract}
We examine Maris' recent suggestion that the fission of electron-inhabited bubbles in liquid helium may give rise to a new form of electron fractionization. We introduce a one-dimensional toy-model - a simplified analogue of the helium system - which may be analyzed using the Born-Oppenheimer approximation. We find that none of the model's low-lying energy eigenstates have the form suggested by Maris' computations, in which the bubbles were treated completely classically. Instead, the eigenstates are quantummechanically entangled superposition states, which the classical treatment overlooks.
\end{abstract}

\footnotetext{
${ }^{1}$ baltschu@mit.edu
} 
Recently, Maris [1] has suggested that the splitting of an electron-inhabited bubble in liquid helium may result in a division of the electron into separate pieces. Each piece would behave like only a fraction of the original electron. This phenomenon would be entirely different from the any of the accepted forms of fermion fractionization [2, 3, 4]. Maris' interpretation has been criticized [5] as corresponding to an ordinary quantum superposition phenomenon and not indicating any fractionization of the electron. We shall discuss this criticism further.

Maris considers an electron trapped in bubble in liquid helium. The repulsive interactions between the single electron and the electron clouds of the helium atoms support the bubble and keep the electron confined in a deep potential well. The electron is initially in the ground (1s) state of the well, before being excited into the 1p state. Maris considers the time evolution of the coupled electron-bubble system after the electron is excited.

Maris treats the electron quantum mechanically and the bubble classically. The electron's $1 p$ wave function is not spherically symmetric, so it exerts different pressures at different points on the bubble. Maris treats this pressure gradient classically and studies its effect on the bubble's shape. He concludes that the bubble will deform and eventually split into two separate bubbles, each containing half of the electron's wave function.

We contend that the classical analysis does not capture the correct time evolution of the bubble's shape. When the entire system is treated quantum-mechanically, the electron's wave function becomes entangled with the wave function of the bubble. The end result is not two bubbles, each containing half of the electron, but rather a system in an entangled state - a superposition of two bubbles in different positions. If the position of the electron is measured, the system will collapse into a state with only a single bubble, surrounding the location where the electron is found.

To shed light on this problem, we shall consider a toy model which is similar to the liquid helium system in many important ways. Our model is one-dimensional and contains only three interacting particles, so we may learn a great deal about the behavior of the system using simple, analytical techniques. We shall make particular use of the Born-Oppenheimer approximation.

The system contains two heavy objects of equal mass $M$ (the "atoms") and a lighter object of mass $m$ (the "electron"), moving on a segment of length $L$ with periodic boundary conditions. The atoms and electron interact through repulsive potentials. If we let $x_{1}$, $x_{2}$, and $x_{e}$ be the positions of the atoms and the electron and set $\hbar=1$, the Hamiltonian is

$$
\begin{aligned}
H & =K+V \\
K & =-\frac{1}{2 M} \frac{\partial^{2}}{\partial x_{1}^{2}}-\frac{1}{2 M} \frac{\partial^{2}}{\partial x_{2}^{2}}-\frac{1}{2 m} \frac{\partial^{2}}{\partial x_{e}^{2}} \\
V & =V_{0}\left\{\cos \left[\frac{2 \pi}{L}\left(x_{1}-x_{2}\right)\right]+2 \epsilon \cos \left[\frac{2 \pi}{L}\left(x_{e}-x_{1}\right)\right]+2 \epsilon \cos \left[\frac{2 \pi}{L}\left(x_{e}-x_{2}\right)\right]\right\} .
\end{aligned}
$$

We shall insist the the potentials be strongly confining; $V_{0}$ must be much larger than the 
kinetic energy scale $\frac{1}{m L^{2}}$. We shall also require that $\epsilon<1$, but we shall assume that it is $\mathcal{O}(1)$.

It is convenient to separate out the center of mass motion and define new coordinates,

$$
\begin{aligned}
X & =\frac{M x_{1}+M x_{2}+m x_{e}}{2 M+m} \\
y & =x_{2}-x_{1} \\
x & =x_{e}-\frac{x_{1}+x_{2}}{2} .
\end{aligned}
$$

In these coordinates, we have

$$
\begin{aligned}
K & =-\frac{1}{2(2 M+m)} \frac{\partial^{2}}{\partial X^{2}}-\frac{1}{M} \frac{\partial^{2}}{\partial y^{2}}-\frac{1}{2 \mu} \frac{\partial^{2}}{\partial x^{2}} \\
V & =V_{0}\left\{\cos \left(\frac{2 \pi}{L} y\right)+2 \epsilon \cos \left[\frac{2 \pi}{L}\left(x-\frac{y}{2}\right)\right]+2 \epsilon\left[\frac{2 \pi}{L}\left(x-\frac{y}{2}\right)\right]\right\} \\
& =V_{0}\left[\cos \left(\frac{2 \pi}{L} y\right)+4 \epsilon \cos \left(\frac{\pi}{L} y\right) \cos \left(\frac{2 \pi}{L} x\right)\right]
\end{aligned}
$$

where $\mu \equiv \frac{2 m M}{2 M+m}$ is a reduced mass for the system.

Writing the full wave function as

$$
\Psi\left(x_{1}, x_{2}, x_{e}\right)=e^{i P X} \psi(x, y),
$$

the Hamiltonian governing the relative motion wave function $\psi$ is

$$
H_{\mathrm{rel}}=-\frac{1}{M} \frac{\partial^{2}}{\partial y^{2}}-\frac{1}{2 \mu} \frac{\partial^{2}}{\partial x^{2}}+V_{0}\left[\cos \left(\frac{2 \pi}{L} y\right)+4 \epsilon \cos \left(\frac{\pi}{L} y\right) \cos \left(\frac{2 \pi}{L} x\right)\right] .
$$

Since $\Psi$ is periodic in $x_{1}, x_{2}$, and $x_{e}, \psi$ must have the "helical" boundary conditions

$$
\begin{aligned}
\psi(x+L, y) & =\psi(x, y) \\
\psi(x, y+L) & =\psi(x+L / 2, y) .
\end{aligned}
$$

Although these boundary conditions mix $x$ and $y$, the entire physical region is contained within the bounds $0 \leq x<L, 0 \leq y<L$; so (11) and (12) do not cause any additional mixing of the $x$ and $y$ dynamics.

We shall analyze the behavior of $\psi$ using the Born-Oppenheimer approximation. This approximation is justified by the existence of two widely separated mass scales, $m$ and $M$. Since $M \gg m \approx \mu$, the atoms move very slowly compared to electron. We may consider $y$ to be an adiabatically varying parameter and solve the Schrödinger equation governing the $x$ motion. The Hamiltonian for the electron's relative motion is

$$
H_{\mathrm{rel} e}=-\frac{1}{2 \mu} \frac{d^{2}}{d x^{2}}+4 \epsilon V_{0} \cos \left(\frac{\pi}{L} y\right) \cos \left(\frac{2 \pi}{L} x\right) \text {. }
$$


We shall distinguish two different parameter regimes. When $\epsilon V_{0}\left|\cos \left(\frac{\pi}{L} y\right)\right|$ is large compared to $\frac{1}{\mu L^{2}}$, the potential $V_{\text {rele }}$ in (13) is strongly confining. However, when $\frac{1}{\mu L^{2}} \gg$ $\epsilon V_{0}\left|\cos \left(\frac{\pi}{L} y\right)\right|$, the electron is nearly free. We must analyze these two cases separately.

We shall assume for now that $y$ lies in the region $0 \leq y \leq L / 2$. The minimum value of $\cos \left(\frac{\pi}{L} y\right)$ occurs when $y=L / 2$. In the vicinity of this point, $4 \epsilon V_{0} \cos \left(\frac{\pi}{L} y\right) \approx 4 \epsilon V_{0}\left(\frac{\pi}{L} \Delta y\right)$, where $\Delta y \equiv L / 2-y$. So the transition between the two regimes occurs when $\Delta y \sim \frac{1}{V_{0} m L}$. Because $\frac{1}{V_{0} m L^{2}}$ is small, $\frac{\Delta y}{L} \ll 1$ in both the weakly confined and transition regions. The potential is strongly confining for all values of $y$, except for in a comparatively small region around $\Delta y=0$.

Let us first consider the strongly confined regime. The potential $V_{\text {rele }}$ has its minimum at $x=L / 2$. Near $x=L / 2, V_{\text {rele }}$ has the form

$$
V_{\text {rele }}(x) \approx 4 \epsilon V_{0} \cos \left(\frac{\pi}{L} y\right)\left[-1+\frac{1}{2}\left(\frac{2 \pi}{L} \Delta x\right)^{2}\right],
$$

for $\Delta x \equiv x-L / 2$. So $H_{\text {rele }}$ becomes

$$
H_{\text {rele }} \approx-\frac{1}{2 \mu} \frac{d^{2}}{d x^{2}}-4 \epsilon V_{0} \cos \left(\frac{\pi}{L} y\right)+\frac{1}{2} \mu\left[\frac{4 \pi}{L} \sqrt{\frac{\epsilon V_{0} \cos \left(\frac{\pi}{L} y\right)}{\mu}}\right]^{2}(\Delta x)^{2} .
$$

This has solutions that are approximately given by harmonic oscillator wave functions $\phi_{n}(\Delta x)$ centered at $\Delta x=0$. The corresponding energies are

$$
E_{\mathrm{rel} e, n} \approx-4 \epsilon V_{0} \cos \left(\frac{\pi}{L} y\right)+\left(n+\frac{1}{2}\right)\left(\frac{4 \pi}{L}\right) \sqrt{\frac{\epsilon V_{0} \cos \left(\frac{\pi}{L} y\right)}{\mu}} .
$$

The second term in (16) is smaller than the first by a factor of $\mathcal{O}\left(\frac{1}{\sqrt{V_{0} m L^{2}}}\right)$, provided $n+\frac{1}{2}$ is $\mathcal{O}(1)$.

Now we consider the $\frac{1}{\mu L^{2}} \gg \epsilon V_{0}\left|\cos \left(\frac{\pi}{L} y\right)\right|$ regime. In this case, the electron is nearly free. The electron states are plane waves subject to the boundary condition (11), and the energies are

$$
E_{\text {rel } e, n} \approx \frac{2 \pi^{2}}{\mu L^{2}} n^{2} .
$$

The ground state corresponds to $n=0$, and the first electronically excited state is the $n=1$ state that is an odd function of $\Delta x$.

If we extend the energy eigenvalues to cover all possible values of $y$ (not just $0 \leq y \leq$ $L / 2$ ) and restrict our attention to the two lowest-lying electronic states, we get

$$
E_{\text {rel } e, n} \approx\left\{\begin{array}{l}
-4 \epsilon V_{0}\left|\cos \left(\frac{\pi}{L} y\right)\right|+\left(n+\frac{1}{2}\right)\left(\frac{4 \pi}{L}\right) \sqrt{\frac{\epsilon V_{0}\left|\cos \left(\frac{\pi}{L} y\right)\right|}{\mu}} \\
\frac{2 \pi^{2}}{\mu L^{2}} n^{2}
\end{array}\right.
$$


The upper expression for $E_{\text {rel } e, n}$ is valid whenever $|y-L / 2| \gg \frac{1}{\epsilon V_{0} \mu L}$ (taking $0 \leq y<L$ ), while the lower is correct if $|y-L / 2| \ll \frac{1}{\epsilon V_{0} \mu L}$. If we include only terms which are zeroth-order in $\frac{1}{V_{0} m L^{2}}$, we have

$$
E_{\text {rel } e, n} \approx-4 \epsilon V_{0}\left|\cos \left(\frac{\pi}{L} y\right)\right|
$$

We obtain this simplified form because the two expressions for $E_{\text {rel } e, n}$ agree near $y=L / 2$ in this approximation (i.e. they both vanish). However, we must still keep in mind that this form for $E_{\mathrm{rel} e, n}$ is not quantitatively accurate near $y=L / 2$.

This yields an effective potential for the relative motion of the two atoms, given by

$$
\begin{aligned}
V_{\mathrm{eff}}(y) & =V_{0}\left[\cos \left(\frac{2 \pi}{L} y\right)-4 \epsilon\left|\cos \left(\frac{\pi}{L} y\right)\right|\right] \\
& =-\left(1+2 \epsilon^{2}\right) V_{0}+2 V_{0}\left[\left|\cos \left(\frac{\pi}{L} y\right)\right|-\epsilon\right]^{2} .
\end{aligned}
$$

$\left[V_{\text {eff }}(y)\right.$ has a cusp at $y=L / 2$, but we have already noted that we expect $E_{\text {rel } e, n}$ to have a slightly different form in the region around this point. This effect should smooth out $V_{\text {eff }}$ in the vicinity of this local maximum.] If $\epsilon<1$, this effective potential has symmetrybreaking minima at $y_{0} \equiv \frac{L}{\pi} \cos ^{-1} \epsilon$ and $L-y_{0}$ (or, equivalently, at $y_{0}$ and $-y_{0}$ ). At these points, $V_{\text {eff }}(y)=-\left(1+2 \epsilon^{2}\right) V_{0}$. If $\epsilon \geq 1$, the electron-atom repulsion overpowers the atomatom repulsion, and the minimum energy configuration has $y=0$; this is possible because the repulsive cosine potential lacks a hard core region. It was for this reason that we required that $\epsilon<1$. We need $\epsilon$ to be $\mathcal{O}(1)$ so that our formula for $V_{\text {eff }}^{\prime}$ is valid at $y=y_{0}$. That is, $y_{0}$ must lie in the regime where the electron is tightly confined; if $\epsilon<\frac{1}{\sqrt{V_{0} \mu L^{2}}}$, then $y_{0}$ will lie too close to $y=L / 2$.

The two minima of $y$ correspond to different arrangements of the system. Since the system is translationally invariant, we may choose to fix $x_{1}=0$, so that $y$ becomes the position of atom 2. If $y=y_{0}$, the minimum of the the electron potential $V_{\text {rele }}$ lies at $x=L / 2$. So the electron will be localized near $x_{e}=L / 2+y_{0} / 2$. Since $0<y_{0}<L / 2$, the order of the particles as we move in the positive direction is atom 1 , atom 2 , electron; because the system is periodic, this is equivalent to atom 2 , electron, atom 1 . If $y=L-y_{0}$, $V_{\text {rele }}$ localizes $x$ near $x=0$, so the electron wave function is peaked at $x_{e}=L / 2-y_{0} / 2$. The order of the particles becomes atom 1, electron, atom 2. In each case, the electron pushes the two atoms apart. The distance from the atom on the electron's left to the one on the electron's right (measured through the electron) is $L-y_{0}>L / 2$.

Since $V_{\text {eff }}(y)$ is a even function of $y$, the exact eigenstates of this potential must be states of definite parity. The parity eigenstates may be constructed as superpositions

$$
\psi(x, y) \approx \frac{1}{\sqrt{2}}\left[\Phi\left(y-y_{0}\right) \phi_{n}(x-L / 2) \pm \Phi\left(L-y_{0}-y\right) \phi_{n}(x)\right]
$$


where $\Phi\left(y-y_{0}\right)$ is an approximate eigenstate of the Hamiltonian $H_{\text {eff }}^{\prime}=-\frac{1}{M^{2}} \frac{d^{2}}{d y^{2}}+$ $V_{\text {eff }}^{\prime}$, localized around $y=y_{0}$. These superpositions are not, strictly speaking, BornOppeneheimer states, but they should be good approximations to the energy eigenstates. The specific form (21) is dependent upon parity invariance, but the general superposition structure is not. For a general (asymmetric) potential with two local minima, the energy eigenstates are superpositions of states localized around those two minima. If $|+\rangle$ is a state located at one minimum and $|-\rangle$ is located at the other, then the eigenstates are $\frac{1}{\sqrt{2}}\left(v_{ \pm}|+\rangle \pm v_{\mp}|-\rangle\right)$; the $v_{ \pm}$are related to the matrix elements of the Hamiltonian by

$$
v_{ \pm}=\sqrt{1 \pm \frac{\delta}{\sqrt{\delta^{2}+4|\langle+|H|-\rangle|^{2}}}}
$$

where $\delta \equiv\langle+|H|+\rangle-\langle-|H|-\rangle$ is the difference in energies between the two minima. $\delta$ is assumed to be small compared to $\langle+|H|+\rangle$ and comparable in magnitude to $\langle+|H|-\rangle$.

The form (21) for $\psi$ holds in the region $0 \leq y \leq L, 0 \leq x \leq L$; it may be extended to other values of $x$ and $y$ using the boundary conditions (11 12). There are no low-lying energy eigenstates for which the mean atomic separation is $L / 2$ that do not have this superposition form, because $y=L / 2$ is a local maximum of $V_{\text {eff }}^{\prime}$.

The two superposition states in (21) are not degenerate. There will be some mixing between the two states, shifting the energy of each. We shall now show that this energy shift is very small, so that the expressions given in (21) are very good approximations to the exact wave functions.

In the WKB approximation, the energy difference between the two states is $\Delta E=$ $\frac{\omega T}{\pi}[6] ; \omega$ is the frequency of classical oscillations about the minima, and $T$ is the tunneling amplitude $T=\exp \left[-\int \sqrt{M\left(V_{\text {eff }}-E\right)} d x\right]$, where the integration extends over the barrier region. The small oscillation frequency $\omega$ is just

$$
\begin{aligned}
\omega & =\sqrt{\left.\left(\frac{2}{M}\right) \frac{d^{2}}{d y^{2}} V_{\mathrm{eff}}(y)\right|_{y=y_{0}}} \\
& =\left(\frac{2 \pi}{L}\right) \sqrt{\frac{2 V_{0}\left(1-\epsilon^{2}\right)}{M}} .
\end{aligned}
$$

Because of the model's periodic boundary conditions, $T$ is actually composed of two terms, $T_{1}$ and $T_{2}$. These correspond to the amplitude for the particle to tunnel to the right (from $y_{0}$ to $L-y_{0}$ ) and for it to tunnel to the left (from $y_{0}$ to $-y_{0}$ ), respectively. If we neglect the quantum fluctuations around the minima, then $T$ is given by

$$
\begin{aligned}
& T=T_{1}+T_{2} \\
& T=\exp \left\{-\int_{y_{0}}^{L-y_{0}} d y \sqrt{M\left[V_{\text {eff }}(y)-V_{\text {eff }}\left(y_{0}\right)\right]}\right\}+\exp \left\{-\int_{-y_{0}}^{y_{0}} d y \sqrt{M\left[V_{\text {eff }}(y)-V_{\text {eff }}\left(y_{0}\right)\right]}\right\}
\end{aligned}
$$




$$
\begin{aligned}
= & \exp \left[-\frac{2 L \sqrt{2 M V_{0}}}{\pi} \int_{\cos ^{-1} \epsilon}^{\frac{\pi}{2}} d u(\epsilon-\cos u)\right]+\exp \left[-\frac{2 L \sqrt{2 M V_{0}}}{\pi} \int_{0}^{\cos ^{-1} \epsilon} d u(\cos u-\epsilon)\right] \\
= & \exp \left[-\frac{2 L \sqrt{2 M V_{0}}}{\pi}\left(\epsilon \sin ^{-1} \epsilon+\sqrt{1-\epsilon^{2}}-1\right)\right] \\
& +\exp \left[-\frac{2 L \sqrt{2 M V_{0}}}{\pi}\left(\sqrt{1-\epsilon^{2}}-\epsilon \cos ^{-1} \epsilon\right)\right] .
\end{aligned}
$$

We conclude that the energy difference between the two states, $\Delta E=\frac{\omega\left(T_{1}+T_{2}\right)}{\pi}$ is an exponentially small function of the large parameter $\sqrt{V_{0} M L^{2}}$, so the mixing between the two superposition states is minimal.

We may now relate our toy model to the liquid helium problem. In each system, the electron repels the atoms surrounding it. The electron-filled bubble in the liquid helium system corresponds to the "bubble" the electron in the toy model creates by forcing apart the atoms on its left and its right.

The states corresponding to (21) in the liquid helium system are superpositions of different position states of the bubble. A single bubble exists at either of two locations; the two spatially separated bubbles do not coexist simultaneously. A classical treatment of the bubble fails to account for these states. The analogue in the toy model of treating the bubble as a purely classical object is the assumption that the wave function must be an unentangled product of a function of $x$ and a function of $y$. Specifically, the two-bubble, split-electron state from [1] corresponds to the toy model state

$$
\psi_{\text {class }}(x, y)=\frac{1}{2}\left[\Phi\left(y-y_{0}\right)+\Phi\left(L-y_{0}-y\right)\right]\left[\phi_{0}(x)-\phi_{0}(x-L / 2)\right],
$$

which is clearly not an eigenstate of the energy.

The analogue of $\psi_{\text {class }}(x, y)$ for the helium system may be written schematically as

$$
\psi_{\text {class }}^{\mathrm{He}}\left(\mathbf{X}_{b}, \mathbf{X}_{e}\right)=\frac{1}{2}\left[\psi_{1}^{b}\left(\mathbf{X}_{b}\right)+\psi_{2}^{b}\left(\mathbf{X}_{b}\right)\right]\left[\psi_{1}^{e}\left(\mathbf{X}_{e}\right)-\psi_{2}^{e}\left(\mathbf{X}_{e}\right)\right] .
$$

$\mathbf{X}_{b}$ and $\mathbf{X}_{e}$ are the coordinates of the bubble and electron, respectively, while $\psi_{i}^{b}$ and $\psi_{i}^{e}$ are appropriate wave functions, localized at two different positions indexed by $i$. It is easy to see that this wave function is not an energy eigenstate. The $\psi_{1}^{b}\left(\mathbf{X}_{b}\right) \psi_{2}^{e}\left(\mathbf{X}_{e}\right)$ and $\psi_{2}^{b}\left(\mathbf{X}_{b}\right) \psi_{1}^{e}\left(\mathbf{X}_{e}\right)$ terms correspond to the electron and the bubble being in different locations. These configurations are unstable; the empty bubble will rapidly collapse, and a new bubble will form around the electron. This process will occur even while the two bubbles are still splitting apart; as the bubble splits, the bubble and electron wave functions will become entangled. For this reason, it is incorrect to treat the bubble classically.

We also note that, if (26) were a stationary state, it would be possible to send fasterthan-light signals between the two bubbles. We would begin by separating the two bubbles 
by a large distance. Then we measure whether or not the electron is present within one bubble. If we find the electron in that bubble, we know that the electron is not present in the other bubble, and the other bubble consequently will collapse. Similarly, if we do not find the electron in the first bubble, it must be in the second bubble. The second bubble with then expand, because it now contains an entire electron. In either case, our measurement has affected the size of the second bubble in a measurable way, so someone observing the second bubble would immediately know that we had performed the measurement 2 . Of course, this paradox does not arise for the superposition wave functions

$$
\psi^{\mathrm{He}}\left(\mathbf{X}_{b}, \mathbf{X}_{e}\right)=\frac{1}{\sqrt{2}}\left[\psi_{1}^{b}\left(\mathbf{X}_{b}\right) \psi_{1}^{e}\left(\mathbf{X}_{e}\right) \pm \psi_{2}^{b}\left(\mathbf{X}_{b}\right) \psi_{2}^{e}\left(\mathbf{X}_{e}\right)\right]
$$

a measurement of the electron's position reveals a full-sized bubble at the location where the electron is found and no bubble at all in the other location.

Finally, we point out that the superposition states displayed in (21) are exactly the sort of states discussed in [5]. If we attempt to measure the fermion number between atom 1 on the left and atom 2 on the right, we find that the expectation value is fractional - with

value $\frac{1}{2}$. However, the presence of the two nearly degenerate superposition states leads to a large dispersion in this localized fermion number. An analogous phenomenon occurs for the liquid helium wave function (27). In each case, the large dispersion indicates that the fractionization is a characteristic of the expectation values only - not of the eigenvalues.

\section{Acknowledgments}

We wish to thank R. Jackiw for many helpful discussions. This work is supported in part by funds provided by the U. S. Department of Energy (D.O.E.) under cooperative research agreements DE-FC02-94ER40818 and DE-FG02-91ER40676.

\section{References}

[1] H. Maris, J. Low Temp. Phys. 120, 173 (2000).

[2] R. Jackiw, C. Rebbi, Phys. Rev. D 13, 3398 (1976).

[3] W.-P. Su, J. R. Schrieffer, A. Heeger, Phys. Rev. Lett. 42, 1698 (1979).

[4] R. B. Laughlin, Phys. Rev. Lett. 50, 1395 (1983).

[5] R. Jackiw, C. Rebbi, J. R. Schrieffer, J. Low Temp. Phys 122, 587 (2001).

\footnotetext{
${ }^{2}$ We would like to thank S. Glashow for pointing out the acausal nature of this situation
} 
[6] L. D. Landau, E. M. Lifshitz, Quantum Mechanics (Pergamon Press, Oxford, England, 1977) p. 167-172, 183-184. 